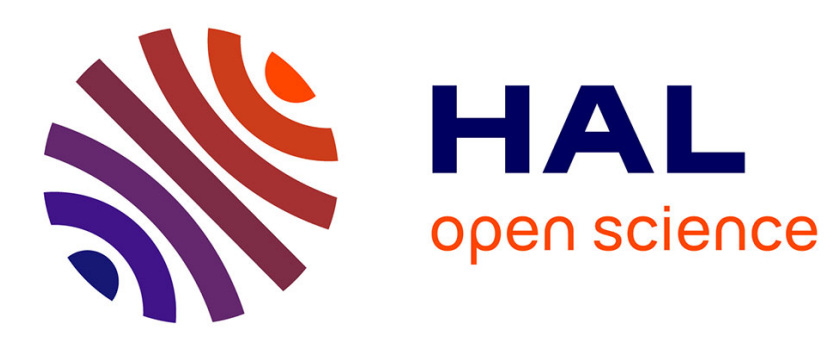

\title{
Dissecting functional connectivity of neuronal microcircuits: experimental and theoretical insights
}

Sarah Feldt, Paolo Bonifazi, Rosa Cossart

\section{To cite this version:}

Sarah Feldt, Paolo Bonifazi, Rosa Cossart. Dissecting functional connectivity of neuronal microcircuits: experimental and theoretical insights. Trends in Neurosciences, 2011, 34 (5), pp.225-236. 10.1016/j.tins.2011.02.007 . hal-01848209

\section{HAL Id: hal-01848209 \\ https://hal-amu.archives-ouvertes.fr/hal-01848209}

Submitted on 27 Aug 2018

HAL is a multi-disciplinary open access archive for the deposit and dissemination of scientific research documents, whether they are published or not. The documents may come from teaching and research institutions in France or abroad, or from public or private research centers.
L'archive ouverte pluridisciplinaire HAL, est destinée au dépôt et à la diffusion de documents scientifiques de niveau recherche, publiés ou non, émanant des établissements d'enseignement et de recherche français ou étrangers, des laboratoires publics ou privés. 


\title{
Dissecting functional connectivity of neuronal microcircuits: experimental and theoretical insights
}

\author{
Sarah Feldt ${ }^{1,2,3,4^{*}}$, Paolo Bonifazi ${ }^{1,2,3,5^{*}}$ and Rosa Cossart ${ }^{1,2,3}$ \\ ${ }^{1}$ Institut National de la Santé et de la Recherche Médicale (INSERM) Unité 901, Marseille, 13009, France \\ ${ }^{2}$ Institut de Neurobiologie de la Méditerranée (INMED), Marseille 13009, France \\ ${ }^{3}$ Université de la Méditerranée, UMR S901 Aix-Marseille 2, 13009, France \\ ${ }^{4}$ Department of Anatomy and Neurobiology, University of California, Irvine, CA 92697, USA \\ ${ }^{5}$ Beverly and Sackler Faculty of Exact Sciences School of Physics and Astronomy, Tel Aviv University, 69978 Ramat Aviv, Israel
}

\begin{abstract}
Structure-function studies of neuronal networks have recently benefited from considerable progress in different areas of investigation. Advances in molecular genetics and imaging have allowed for the dissection of neuronal connectivity with unprecedented detail whereas in vivo recordings are providing much needed clues as to how sensory, motor and cognitive function is encoded in neuronal firing. However, bridging the gap between the cellular and behavioral levels will ultimately require an understanding of the functional organization of the underlying neuronal circuits. One way to unravel the complexity of neuronal networks is to understand how their connectivity emerges during brain maturation. In this review, we will describe how graph theory provides experimentalists with novel concepts that can be used to describe and interpret these developing connectivity schemes.
\end{abstract}

\section{Introduction}

More than 20 years ago, Miles and Wong first made a puzzling and seminal observation: stimulating a single neuron could trigger network synchronization in disinhibited hippocampal slices [1]. A number of recent studies have similarly reported that stimulation of single neurons can affect population activity in vitro as well as in vivo [2-9] (Figure 1). However, there are only a handful of such reports, probably because neurons with the ability to influence network dynamics are rare and/or conditions under which one can observe such phenomenon are specific. Regardless, the direct impact of single neurons on network and behavioral outputs demonstrates the importance of the specific structural and functional organization of the underlying circuitry $[2,7,8]$. At this point, the next important step is therefore to understand how specific network structures can empower single neurons to govern network dynamics.

This is a timely issue because the neuroanatomical description of network structure is currently experiencing a new era, fed by recent advances in molecular genetics and light microscopy [10,11]. Novel methods such as optogenetics, heterologous receptor expression and retrogradely transported viral vectors allow both trans-synaptic circuit analysis in addition to targeted and precise control of single-

\footnotetext{
Corresponding author: Cossart, R. (cossart@inmed.univ-mrs.fr)

These authors contributed equally to this work.
}

cell firing [12-16]. Parallel to the considerable advances in the description of neuronal network structure, the past decade has seen the emergence of powerful in vivo experimental strategies [17-23] to study how brain function is encoded in the firing of neuronal assemblies. Although in vivo analysis clearly demonstrates that brain functions might be encoded by specific neuronal activity patterns with characteristic temporal dynamics, information is often still lacking on the detailed neuroanatomical structure of the microcircuits activated during a particular behavioral task [24].

How can the gap between the in vivo exploration of function and fine neuroanatomical description of structure be filled? Complex network theory, a new field of theoretical studies that combines graph theory (structure) and complex systems (dynamics), provides neurobiologists with a framework to interpret structure-function relationships in neuronal networks. Therefore, in this review, we first introduce basic notions of network topology and connectivity to provide shared common definitions to the different areas of expertise. Next, we review applications of complex network theory to neurobiological questions, in particular, by analyzing structure-function relationships in the field of cortical development. We propose that network development provides an interesting and unique environment to dissect how microcircuits are organized to produce function. Because different functional microcircuits tend to develop sequentially, network development offers experimentalists successive temporal windows to observe the impact of individual microcircuits as they develop and give rise to different network dynamics. The application of graph theoretical concepts to these sequential periods allows one to link the structure and function of each microcircuit throughout development. Because, in many aspects, immature networks prefigure the end wiring map of adult circuits, such analyses should also ultimately reveal information about the final organization of mature neuronal networks.

Describing structure-function relationships in neuronal networks from a theoretical perspective

Complex network theory: definitions

Network theory has recently gained much attention through its ability to describe and quantify social, technological 


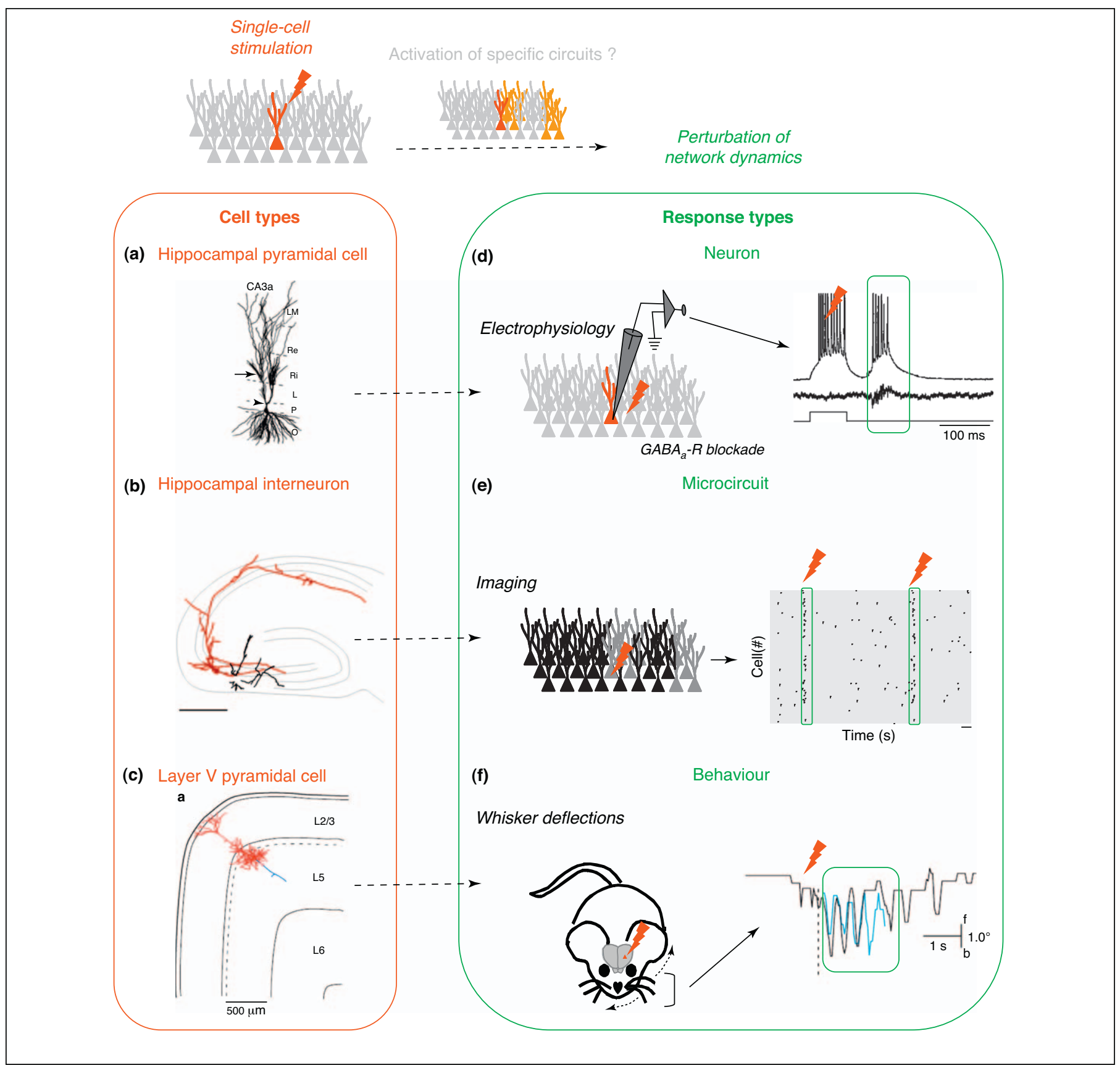

Figure 1. Network effects derived from the action potentials of a single neuron. Inducing action potentials in a single neuron can have effects at the level of network

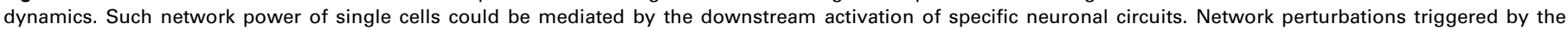

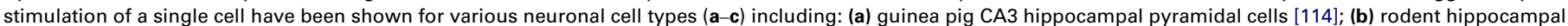

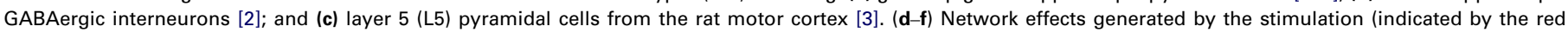
lightening symbols) of these different cell types were measured at different levels of observation: (d) neuronal, (e) microcircuit and (f) the behavioral level using different experimental approaches: in vitro electrophysiology, in vitro imaging and in vivo monitoring of whisker deflections, respectively. (d) Stimulating a single CA3 pyramidal cell in the pacemaker region of disinhibited adult hippocampal slices (i.e. in the presence of the GABA $\mathrm{A}_{\mathrm{A}}$ receptor antagonist picrotoxin) triggered a population burst (green box) measured with intracellular and extracellular electrophysiological recordings [1]. (e) Stimulating a single GABAergic hub neuron in immature hippocampal slices triggered neuronal synchronization (green boxes) as revealed by the rasterplot representing the onset of calcium events from individual cells as a function of time. (f) Intracellular

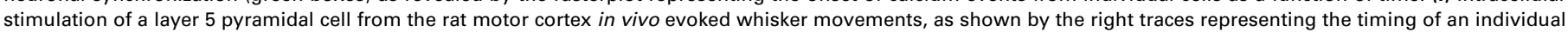
forwards (f) and backwards (b) whisking movement relative to the stimulation [3]. The two traces (black and blue) represent the response to different trials of the same stimulation and show the similarity of the evoked whisker deflection (seen in the green box). Reproduced, with permission, from [114] (panel a), [2] (panels b and e), [3] (panels $\mathbf{c}$ and $\mathbf{f}$ ) and [1] (panel d).

and biological systems. In general, much work in network science focuses on the structure of the network and how this can give rise to various functions/dynamics. For example, the structure of social networks has been used to predict the spread of disease through society $[25,26]$. Furthermore, food webs have been created to understand predator-prey interactions $[27,28]$, the World Wide Web provides a fascinating network on which to study information flow, and multiple algorithms have been developed to detect communities within networks based on a variety of different metrics [29-32]. In this framework, a network is defined by nodes (vertices), which are the elements of the network, and links (edges) connecting the nodes and defining structure. Once the network structure of a system has been obtained, many 
metrics are used to quantify its properties. We will briefly discuss a few of these properties below (see Box 1 for specific examples and [33] for a detailed review).

One of the most commonly described properties of a network is its degree distribution. The degree $(k)$ of a node is defined as its total number of links to other nodes in the network, and it is often used to distinguish between different types of network structures (Box 2). The degree distribution of a network $P(k)$, is the probability distribution that describes the probability of a randomly selected node to have a given degree, $k$. Traditionally, graph theory focused on the study of regular networks (networks built on a lattice in which every node is connected to its $k$ nearest neighbors) or random networks (networks in which each pair of nodes is connected with a probability, $p$, giving rise to a Poisson degree distribution). However, real-world networks tend to fall into neither of these categories, and we will discuss a few examples of networks with degree distributions commonly seen in biological systems.

Many real-world networks are characterized by a power law degree distribution and thus referred to as scale-free networks due to their lack of a characteristic connectivity scale. In other words, by randomly picking a node and finding its degree, one will not be able to say anything about the degree distribution of the network due to the very large variability. An especially interesting feature of these networks is that due to their heavy tailed degree distribution, a small percentage of nodes (called hubs) will be highly connected, whereas most nodes will have a low degree of connectivity. Importantly, hub nodes have been shown to be central with respect to network synchronization and susceptibility to attacks [25,26]. Specifically, scale-free networks have been shown to be robust against random failure of nodes, but targeted attack of hub nodes leaves the network fragmented and severely disrupts information flow. This is clearly seen when examining the Internet, which functions despite the occasional failures of a router, whereas attacks of hub routers cause significant problems [34].

Although many networks display a power law degree distribution, care must be taken when fitting data to such a function because improper classification leads to erroneous

\section{Box 1. Parameters used to estimate basic features of the topology (architecture) of a network}

A number of measures have been defined to describe the properties of network topologies (degree distribution, network size, etc.). A few of the most commonly computed metrics, in addition to examples of software used to analyze network properties, are presented below.

\section{How many connections does a node have?}

Node degree $(\boldsymbol{k})$ The degree $(k)$ of a given node corresponds to the total number of its links (connections), i.e. the number of its nearest neighbors (Figure l, left panel). Different node degree distributions, such as Poisson, exponential and power law define different classes of networks.

\section{How distant are nodes?}

Shortest path length (I) Given a pair of nodes, the shortest path length $(I)$ is the minimal number of links which connects them (Figure I, left panel). The shortest path length introduces a distance between nodes that does not have any spatial meaning. Whereas the total number of nodes $(N)$ defines the size of the network, the shortest path lengths estimate the linear size of the network, i.e. its compactness. The characteristic path length of the network, $\bar{l}$, is the average value of shortest path lengths for all pairs of nodes in the network.

\section{Are neighbors connected?}

Clustering coefficient $(C)$ Given a node with a degree $k$, the clustering coefficient measures the fraction of connections between its $k$ nearest neighbors (Figure I, right panel). The total number of possible connections is $[k(k-1) / 2]$. The clustering coefficient is used to estimate the density of connections 'locally' between groups of neurons that share common nearest neighbors. The clustering coefficient of the network, $C$, is the average value of clustering coefficients for nodes in the network.

\section{How can I easily compute measures of network topology?}

Existing software For those who do not wish to write their own code to analyze data, many stand-alone programs exist for network analysis (e.g. Pajek, Gephi, Cytoscape, etc.). There are also many packages/toolkits available that allow one to implement measures of network topology in various languages: MatlabBGL, Brain Connectivity Toolbox (Matlab), NetworkX (Python), JUNG (Java), igraph (C++, $\mathrm{R}$, Python, Ruby), etc. (Note: these are merely examples and other software also exists).

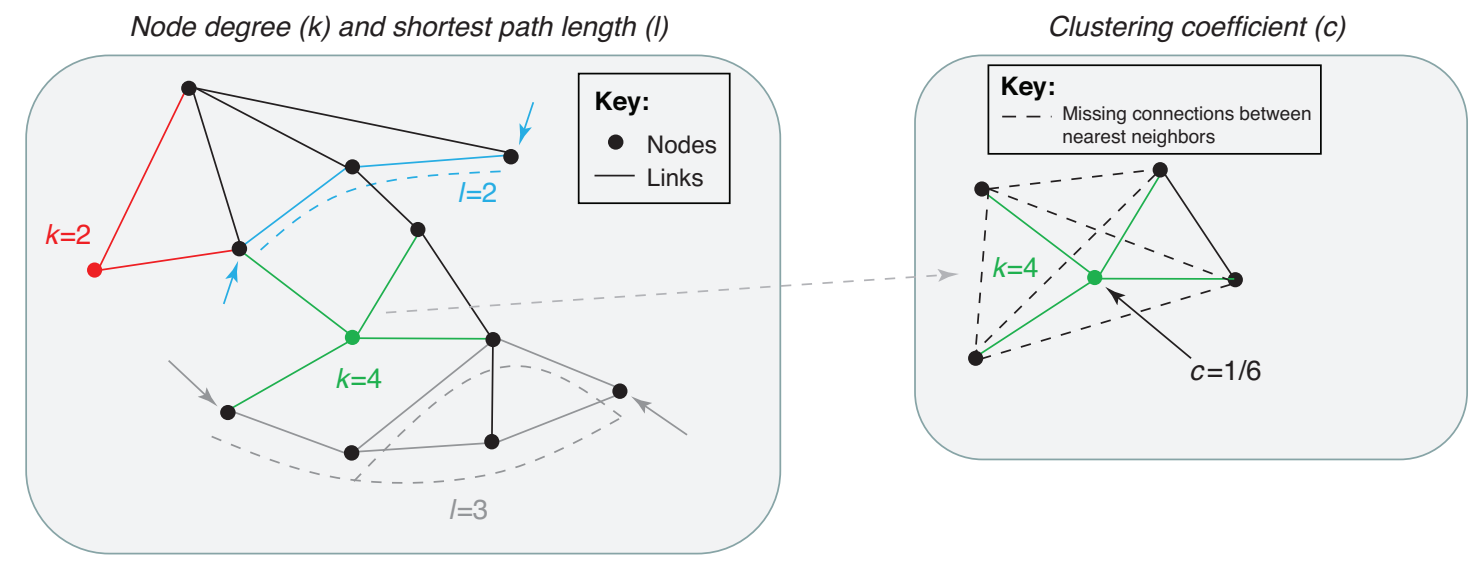

Figure I. Representations of networks as graphs. Left panel: In this schematic example of a simple network, the degree of the green and the red nodes is given by their number of connections, $k=2$ and $k=4$, respectively. The distance (/) between the two nodes marked by blue arrows is 2 (i.e. at least two links separate them). The shortest path is highlighted by the dashed blue line. For the nodes marked by gray arrows, $l=3$. Note that two possible shortest paths exist (indicated by the dashed gray lines). Right panel: In this example, the green node has four nearest neighbors $(k=4)$ and just one connection between them (black solid line) out of six possible ones. The five potential missing connections are marked with dashed lines. The clustering coefficient is $c=1 / 6$. 
Scale-free networks The probability that a node has $k$ connections is described by a power law $\left(p(k) \sim k^{\gamma}\right)$. Since the degree, $k$, of the nodes can span through many orders of magnitude, scale-free networks lack a characteristic scale (note that Poisson random networks have a very clear scale defined by their average connectivity degree). Scale-free networks include hubs, i.e. rare super-connected nodes that can have a strong impact on the global dynamics (indicated by the red node in Figure la). Many real networks have been shown to have scale-free properties with $\gamma$ between 1 and 3 .

Regular networks In a regular network, all of the nodes have the same number of nearest neighbors, and the network has an ordered arrangement (see Figure 1b for an example of a lattice with degree $k=$ 4). Regular networks have a high density of connections between neighbors and therefore are characterized by high clustering coefficients. The characteristic path length, $\bar{l}$, increases as a power of the number of nodes $N$.

Random networks Classically, random networks are obtained by distributing a total of $m$ links to $N$ nodes (Figure lb). As a result of the random process, nodes can have different degrees and the probability that a node has $k$ connections is represented by a Poisson distribution where $k=m / N$ is the average degree of the network. Therefore, they are called Poisson random networks (or Erdos-Renyi graphs after the graph theorists who pioneered their studies). In contrast to regular networks, the characteristic path length, $\bar{l}$, increases logarithmically (i.e. slowly) with the total number of nodes $N\left(\bar{I}_{\text {regular }}>\bar{I}_{\text {random }}\right)$. Random networks lack an abundance of local connections, therefore they have a small clustering coefficient.

Small-world networks Small-world networks share features with regular and random networks (Figure lb). In fact, small-world networks have dense local connections like regular networks $\left(\mathrm{C}_{\text {small- }}\right.$ world $\sim \mathrm{C}_{\text {regular }}$ ) and short characteristic path lengths like random networks ( $I_{\text {small-world }} \sim I_{\text {random }}$ ). Small-world networks can be obtained by introducing a few random connections within a regular network. These random connections do not interfere with the local connectivity of the network (i.e. with the clustering coefficient) but shorten the distance between previously distant nodes (i.e. reduce the characteristic path length). Many real networks have been shown to have small-world properties. (a)
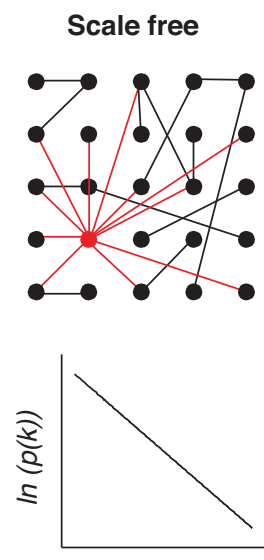

$\ln (k)$

$p(k) \propto k^{-\gamma}$ (b)

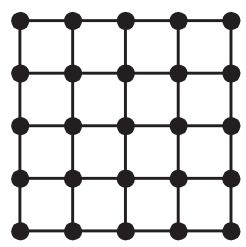

Small-world

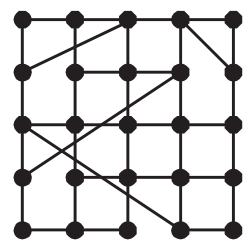

Random
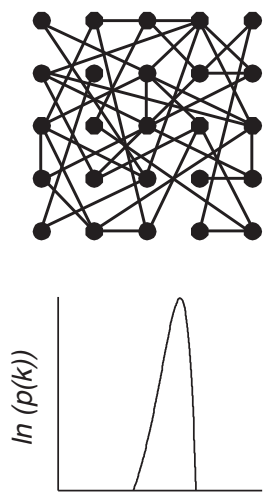

$\ln (k)$

$p(k) \propto \frac{e^{-\underline{k}} \underline{k}^{k}}{k !}$

Figure I. The two main network topologies commonly found in biological systems are (a) scale-free and (b) small-world. Small-world network structure lies between that of regular (left) and random (right) networks.

data interpretation [35]. Accurate fitting and validation of power law distributions is an active area of investigation in statistics. The common practice of plotting the data on a $\log -\log$ scale and attempting to fit it with a straight line has been shown to be poor in correctly distinguishing power law distributions. Methods based on goodness-of-fit and maximum likelihood [36] should be employed to: (i) establish the robustness of the power law fit compared with other functions; and (ii) properly estimate the value of the power law scaling exponent. Furthermore, experimental limitations introduce finite-size effects on power law validations, and neurobiological data samples often only span a few orders of magnitude, which can lead to errors in classification of the underlying degree distribution. For a detailed discussion of how to correctly use statistics when fitting data to a power law, please refer to [36] and the references within.

Another way to describe a network is to quantify its size using the characteristic (average shortest) path length. The shortest path length between two nodes is given by the minimal number of links connecting the nodes. The characteristic path length of the network, $\bar{l}$, is the average value of the shortest path length between all pairs of nodes in the network. Another commonly calculated feature of network structure is the clustering coefficient. For a given node, the clustering coefficient is given by the fraction of existing connections out of all possible connections between the nearest neighbors of the node (Box 1). The clustering coefficient of the network, $C$, is this value averaged over all nodes in the network. Networks with a high number of local connections between nodes, such as regular networks, have a high clustering coefficient. To avoid confusion, it is important to note that these measures calculate distance in units of links between nodes, which do not take into consideration the spatial placement of nodes. Therefore, the nearest neighbors, that is nodes directly connected by one link, can be physically very distant.

The previous measures relate to the second type of network commonly found in real world systems, namely small-world networks. Small-world networks [37] are 
characterized by a high clustering coefficient (such as regular networks) and a small characteristic path length (such as random networks) that increases logarithmically with the number of nodes. A common way of building a small-world network is to start from a regular network where each node is connected to its neighbors within a radius, $r$, giving the network high local clustering. Connections are then randomly rewired with a probability, $p$ [37] (Box 2). The addition of only a few rewired connections introduces global shortcuts, giving the network a small characteristic path length while not disturbing the local connectivity, i.e. the high clustering coefficient. It is important to note that the rewired connections let information flow quickly, thus play- ing a similar role to hubs in scale-free networks regarding network dynamics. An estimation of how much a specific link or node can affect the information flow in a network is given by its 'centrality', which measures the number of shortest paths that pass through it [38].

\section{Complex network theory applied to the description of neuronal networks}

Neuronal networks fit naturally into the framework presented above. Graph theoretical analysis has been applied predominantly to describe neuronal networks in studies examining connection patterns between brain areas at macroscopic scales in the cat cerebral cortex and monkey [39] as

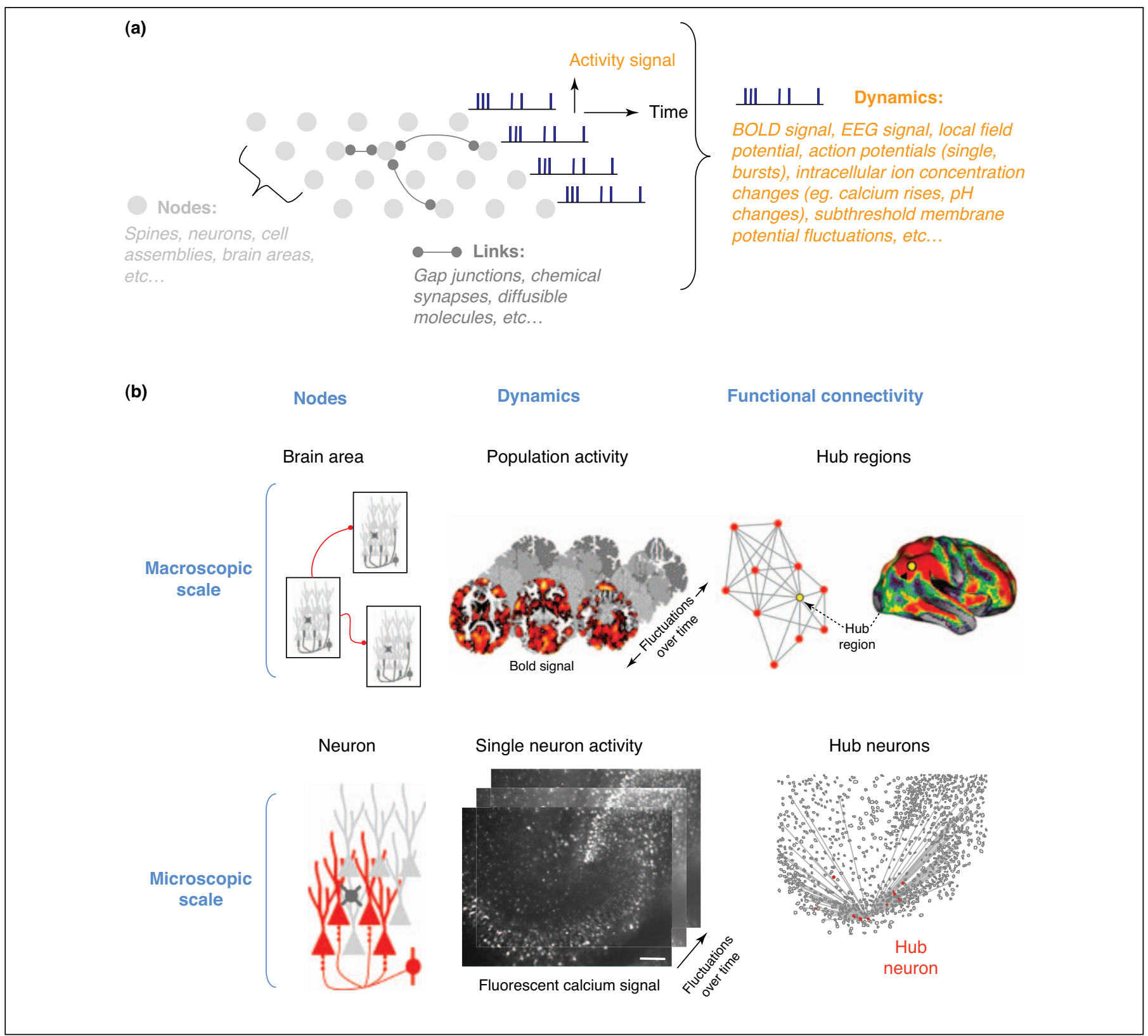

Figure 2. Complex network theory applied to the analysis of networks of neurons. (a) Schematic representation of a neuronal network where nodes can represent different neuronal entities (e.g. spines, cell assemblies, etc.) and links can represent different communication methods (e.g. gap junctions, chemical synapses, etc.). Dynamics characterize the onsets/offsets of a given signal measured for each node as a function of time and can include action potentials or BOLD signals observed with fMRI. (b)

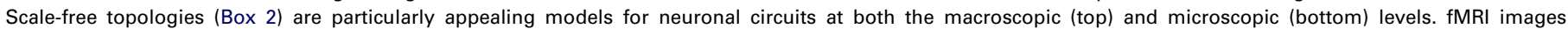
reproduced, with permission, from [115]. Top panel: fMRI allows for the mapping of correlated activity between distant brain regions from which a functional connectivity network can be calculated. Candidate hubs are those regions with disproportionately high connectivity. Bottom panel: Multineuron calcium imaging enables functional connectivity to be described at the scale of microcircuits. It also enables the identification of hub neurons (red), which are functionally connected to many others (functional links in gray). Images and contour map reproduced, with permission, from [2]. 
well as in studies that analyze functional magnetic resonance imaging (fMRI) or electroencephalogram data from the human brain [40-42] (Figure 2). However, only recently has it begun to be employed to describe neuronal microcircuits by experimental neurophysiologists [43]. In fact, the exact implementation of graph theoretical methods will depend on the scale of the investigation, i.e. nodes are represented by single neurons, neuronal assemblies or brain areas (Figure 2). To avoid confusion, it is also important to specify the nature of the connections that are established between the nodes because they can derive from structural (e.g. synaptic links) or dynamical observations (e.g. spike correlations). In agreement with previous definitions $[33,41,44]$, neuronal networks are mainly described by three different types of connectivity reflecting three parallel levels of investigation: anatomical, functional and effective (Box 3).

Anatomical connectivity indicates a physical connection (e.g. synaptic or electrical) between nodes (Box 3). Functional connections indicate a statistical dependence between the activities of two nodes, i.e. a relationship between the activities of two nodes without any assumption on how these correlations are mediated (Box 3). Two neurons are functionally connected if we can predict the firing of one based on the other (e.g. measuring their average firing rate or spike trains). CA1 hippocampal pyramidal cells are known to be functionally connected to basket cell interneurons during sharp wave associated ripples because both cells tend to fire action potentials at the maximal amplitude of the ripple episodes. By contrast, these same pyramidal cells functionally connect with Oriens-Lacunosum Moleculare interneurons during theta oscillations [45]. Functional connectivity can also be extracted from correlations between subthreshold activities, for example, by analyzing membrane potential dynamics. In fact, behavioral modulation of the correlation between membrane potential dynamics of different interneurons and pyramidal cells was recently established in the barrel cortex of awake-behaving mice using multiple whole cell recordings [19]. Therefore, functional connectivity is modulated by behavioral and brain states.

\section{Box 3. The different types of connectivity used to describe neuronal networks}

There are three main types of connectivity used to describe neuronal networks, which reflect three parallel levels of investigation. Descriptions of each of these connections are given below.

Anatomical connectivity indicates physical connections (i.e. chemical synapses, electrical synapses, etc.) between nodes (Figure la).

Functional connectivity indicates a statistical dependence between the activities of two nodes without any assumption of the mechanism by which these relationships are mediated. Two neurons are functionally connected if we can predict the firing of neuron $A$ based on the firing of neuron $B$. Because functional connections do not imply causal relationships, if the activation of two cells is temporally correlated, this does not mean that: 1) the activation of neuron $A$ is required for the activation of neuron $B$; or 2 ) stimulating the firing of neuron $A$ will trigger firing in neuron $B$. The raster plot (Figure $\mathrm{lb}$ ) represents two distinct populations of functionally connected neurons (red and green). Here, connectivity is determined by the synchronous firing of neurons within each population.

Effective connectivity indicates the direct influence that a node exerts on another and, in the context of neuronal circuits, a causa relationship between the activities of two nodes. For example, the firing of one neuron can lead to the activation of another neuron through a direct monosynaptic contact or a polysynaptic path. Different forms of causal modulation (inhibition, phase modulation, firing rate change, etc.) can be envisaged. Effective connectivity can be revealed by perturbing the activity of one neuron and measuring the change in the activity of the other neurons. This is illustrated in Figure Ic by the polysynaptic response (postsynaptic potentials, PSPs) triggered by stimulation of a presynaptic neuron. (a)

Anatomical connectivity

(b)

Functional connectivity
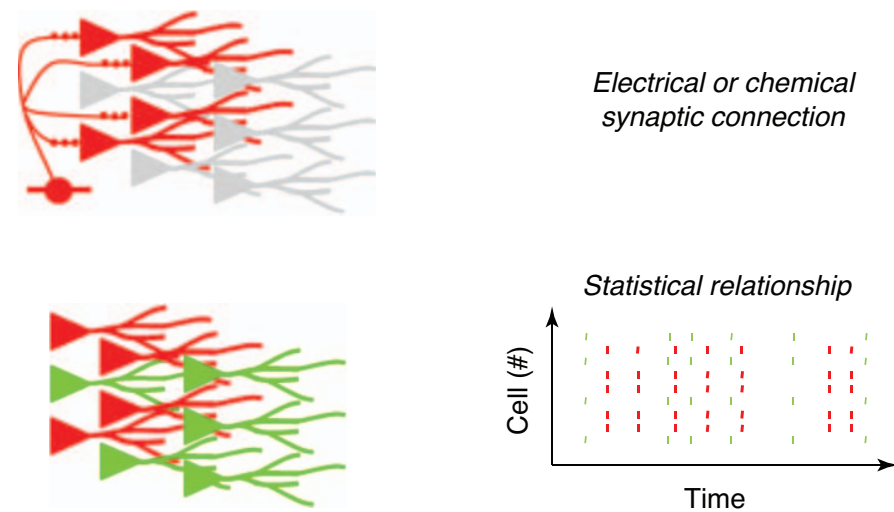

Causal relationship

Effective connectivity

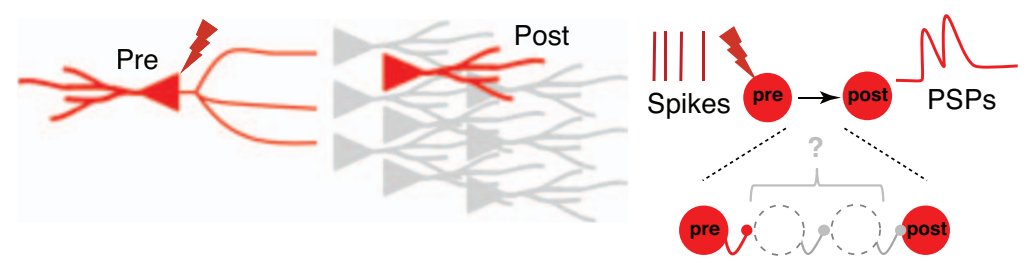

Figure I. Schematic illustration of the three main categories of connectivity described in neuronal networks: (a) anatomical, (b) functional and (c) effective. 
Effective connectivity was first described to be the 'minimal neuronal circuit' that could give rise to the observed dynamics [46], but today the term is generally used to indicate the direct influence that one node exerts on another [47]. More specifically, in the context of neuronal circuits, effective connectivity shows a causal relationship between the activities of two nodes (Box 3). For example, two neurons are effectively connected if the activation of one neuron is able to trigger the firing of the other, without any assumption on how this effect is mediated (e.g. monosynaptic, polysynaptic, etc.). Two neurons that are effectively connected might not be linked by a direct monosynaptic contact because the firing of one cell can lead to the reliable activation of another due to the initiation of a sequence of cellular activations. For example, quadruple electrophysiological recordings from neurons in slices of human neocortex have shown that single action potentials in presynaptic pyramidal cells almost always initiated a chain of polysynaptic events mediated by the sequential activation of specific sets of neurons. Consequently, most pairs of pyramidal neurons in the human neocortex are effectively connected to each other via the intermediate activation of several neuronal partners, including GABAergic interneurons [7]. In addition, an effective connectivity between neurons $A$ and $B$ does not necessarily imply that activating neuron A triggers activation of neuron $B$ because other forms of modulation (such as inhibition or phase changes) of the activity of neuron B can be envisaged. Therefore, although effective connectivity can imply anatomical connectivity, this is not necessarily the case.
When defining anatomical, functional or effective connectivity, links between neurons can be either binary (existent or not), undirected (bi-directional), directed (uni-directional) or weighted (the strength of the connection is included). Most metrics were originally designed for binary networks, but many can also be adapted for weighted and directed networks. However, most network measures are undefined in the case of negative weights, which can present problems for neuroscientists attempting to describe inhibitory connections or anti-correlated firing patterns. In addition, the ease of working with binary networks has sometimes led to the use of thresholding methods to define neuronal connections in a binary way with the drawback of an arbitrary choice of threshold.

Much theoretical and computational work has studied the important relationship between network topology and dynamics [33,48-55]. For example, consider a network of neurons built on a lattice with regular (local), random (global) or small-world connectivity. Given a fixed set of neuronal parameters and network conduction delays, when a local stimulation is applied in a network with regular (local) connectivity, activity travels as waves. However, the same stimulation induces synchronous bursting in a network with random connections and a range of intermediate dynamics in networks with small-world properties [56,57]. The ability of different anatomical network structures to produce different types of dynamics suggests that different anatomical connectivities might give rise to different types of network functionality (as shown in Figure 3). This phenomenon might largely account for

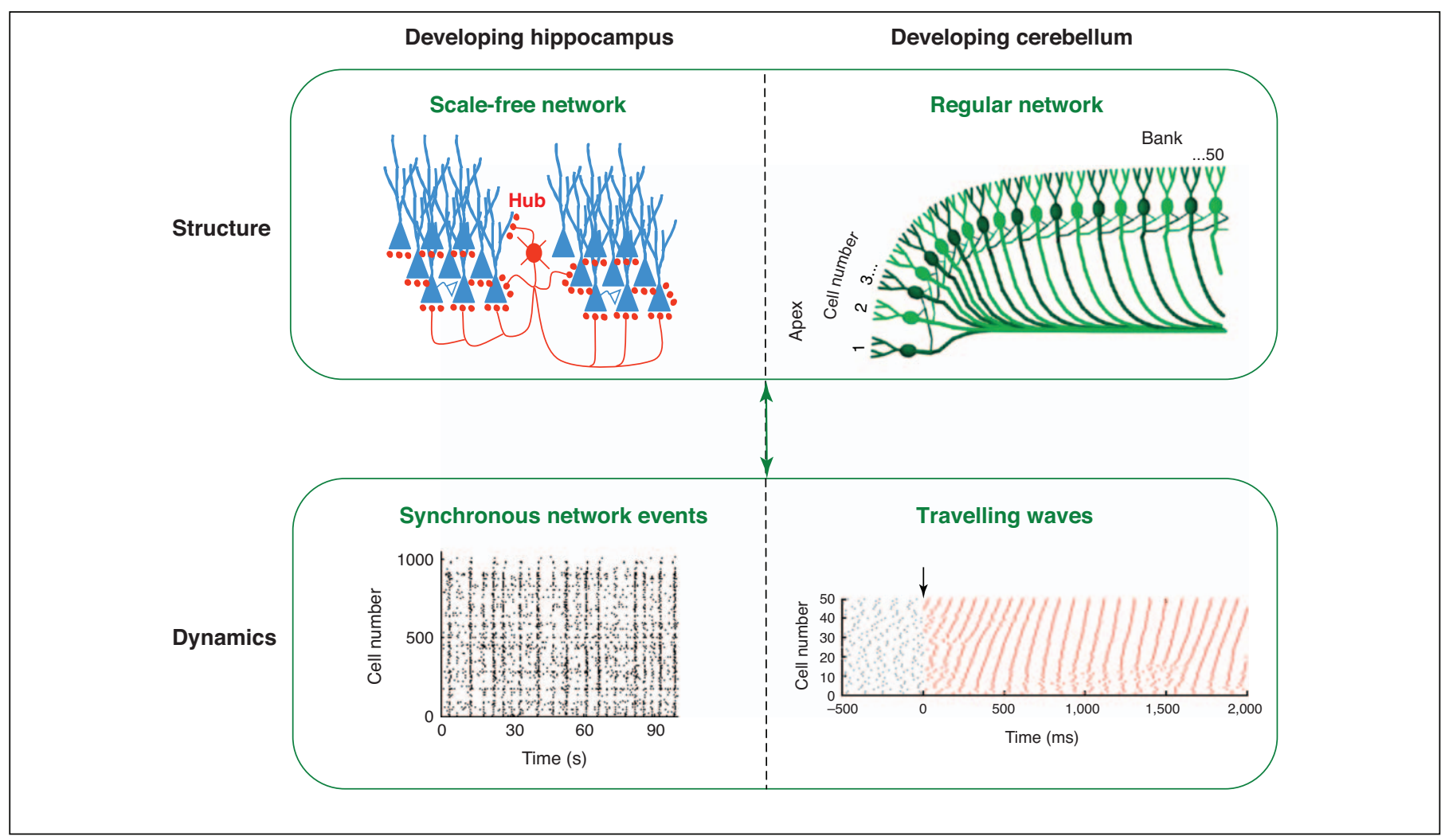

Figure 3. The relationships between structure and dynamics in neuronal networks. The developing hippocampus (left panels) displays synchronous network events (called giant depolarizing potentials) and has a scale-free functional structure [2]. However, the structure of the developing cerebellum (right panels) has been shown to have a regular network structure. In this case, network activity takes place as travelling waves [58]. Adapted, with permission, from [2] and [58], respectively. 
the different network dynamics observed between the developing hippocampus, which displays a scale-free type of functional structure producing synchronous network bursts [2], and the developing cerebellum, which presents a regular organization lattice supporting travelling waves of activity [58].

Small-world and scale-free topologies are particularly appealing models for neuronal circuits [48,51,59-61] (Figure 2). Small-world networks of neurons have been suggested to be a compromise between optimal wiring cost (local networks) and synchronization needed to maintain proper brain function (random networks) [48]. In addition, it has been proposed that in scale-free neuronal networks, neuronal hubs orchestrate behaviorally-relevant activity in cortical assemblies as well as being causal in producing pathological oscillations [51,61-63]. It has recently been shown experimentally [2] that the functional connectivity of developing rodent hippocampal slices presented a scalefree topology that comprised highly connected hub GABAergic neurons orchestrating synchronous activity. Interestingly, GABAergic hub neurons in the developing hippocampus combine a high degree of functional, effective and structural connectivity because stimulating them induces the activation of many neurons, owing to their widespread axonal arborization [2] (Figure 1b). However, it is also worth mentioning that connectivity analysis fed by graph theory most often does not take into account actual metric distances between cells: if the complexity of different cell types is increasingly considered, their distribution in a three dimensional brain is not taken into account (but see [64]). Hence, the notion of 'length' or 'distance' between cells refers to the number of links that separate them $[33,44,65]$ rather than to their actual physical distance (Box 1). For example, if two neurons are always active together in two distinct brain regions (such as the thalamus and the neocortex), their network distance will be short. Measures and methods of analysis designed to take this important spatial information into account have recently been introduced and described in the context of neuronal circuits and macroscopic brain networks $[66,67]$ and will be important to further develop such methods.

The main limitation in applying graph theory tools to describe structure-function relationships in mammalian neuronal networks stems from the fact that such an analysis not only requires simultaneous access to behavior and neuronal activity (which is now possible in light of the development of novel in vivo approaches such as the recent extension of the patch clamp technique, widely used in slice preparations, to freely moving [20] or awake head fixed [21] rodents performing a behavioral task), but also sampling from many neurons repetitively active for statistical relevance. It is interesting to point out that studies using model systems, such as zebrafish [68], Caenorhabditis elegans [35,69] and Drosophila [70], have started using advanced data mining and analyses that are currently far ahead of mammalian brain studies. The reason for this is probably due to the fact that access to entire and large networks can be achieved in the case of these model organisms because of their small sizes and relative architectural simplicity, while at the same time offering a rich behavioral repertoire [68-72]. In fact, it is very probable that many principles of operation of the simpler microcircuits of these microorganisms can be extended to the dissection of more complex nervous systems.

Another approach to circumvent the current limitations of applying graph theory in the adult mammalian brain is to study how neuronal networks develop and form connections initially. In the next section of the review, we will discuss recent data obtained from the developing mammalian brain as examples of how developing neuronal networks might help to reveal the organization of adult neuronal connectivity.

\section{Emergence of structural and functional connectivity during development Developing neuronal networks help reveal the organization of adult neuronal connectivity}

For several reasons, the study of developing neuronal networks provides conceptual and experimental tools to understand structure from a functional perspective, even in adult systems. First, in many ways, immature networks prefigure the fully developed wiring map of adult circuits because several lines of evidence indicate a strong genetic predetermination at early embryonic stages [73]. For example, it was recently shown that synapses develop specifically among sister excitatory neurons in the neocortex [74]: glutamatergic neurons issued from the same progenitors tend to wire together and form neuronal assemblies. This strong genetic predetermination is perhaps even more striking for the cortical GABAergic interneuron population because the morpho-physiological phenotype of adult interneurons is largely predetermined by their spatio-temporal embryonic origins [75]. Hence, the way GABAergic neurons connect into functional mature networks is largely predetermined by their time and place of birth [76]. Second, developing neuronal structures are particularly well suited to structure/function studies because they display simple stereotyped network dynamics [77-81]. In the cortex, neuronal activity is associated with an intracellular calcium rise that can be easily measured with optical approaches [82-85]. Correlated neuronal activity patterns in developing cortical networks have several additional advantages: (i) they are recurrent in the timescale of seconds [77-79], which allows one to sample the same sequence of neuronal activation many times and therefore reach statistical significance in the calculation of functional connectivity maps; (ii) they are relatively easy to monitor experimentally as they are similarly observed in vivo [8589] and in vitro [82-84]; and (iii) they have important functional properties because they participate in network maturation. Hence, developing neuronal networks start functioning while in construction, but their function (producing coordinated activity patterns) is very basic and therefore easier to study than in the mature nervous system where multiple and varied functions have been established.

Although experimental investigation in living developing neuronal networks offers great possibilities for understanding structure-function relationships, most structure-function studies so far have analyzed networks using neurons growing in cultures and not in vivo 
[50,55,90-92]. Indeed, cultures are relatively easy to manipulate and record from, and therefore seem to be a more attractive model for many researchers interested in studying systems neuroscience. Paradoxically, still little is known about the morpho-physiological cellular organization of the developing cortex for several reasons. Perhaps the most critical limitation stems from the fact that developing neurons are not easy to classify into cell types because they have not reached their mature characteristic features [75]. In addition, the links between immature neurons are not only less developed, but also most often radically different from adult ones in many important ways. Some of these differences include: (i) synapses can be silent during early stages of development [93]; (ii) gap junctions are abundant and mediate neuronal synchronization [79,94,95]; (iii) GABA, the main inhibitory transmitter in the adult brain, is depolarizing at early stages in development as a result of higher intracellular chloride concentrations [96]; (iv) tonic currents provide membrane depolarization [77,97-99]; and (v) action potentials are often immature and neurons have a higher tendency to be active in bursts due to different intrinsic properties [80]. In other words, developing cortical networks are not just smaller and poorly connected adult circuits but are very different entities in many ways, and these limitations need to be kept in mind when extrapolating any data obtained from developing networks to the functional connectivity of mature neuronal networks.

What have we learned so far from the study of developing cortical networks regarding structure-function relationships? At macroscopic scales, recent studies demonstrate a strengthening of the functional/structural connectivity relationship as a function of age in the developing human brain [100,101]. At the microcircuit scale, it has been shown using a multidisciplinary approach combining multineuron calcium imaging with online reconstruction of functional connectivity (Figures 1 and 2) that the functional connectivity of the developing hippocampus is scale-free, which implies the existence of high connectivity hub neurons [2]. Calcium imaging of neuronal activity in living brain slices led to the determination of functional network connectivity and the online detection of hub cells. These hub neurons were then targeted for electrophysiological recordings to characterize their morphological properties and probe their network interactions (structural, functional and effective). Importantly, hub neurons combine a high degree of functional, effective and structural connectivity as they display widespread axonal arborizations associated with many direct postsynaptic contacts. Moreover, these hub cells were shown to be GABAergic because they were found to be labeled with green fluorescent protein (GFP) in mice expressing a GFP construct under the glutamic acid decarboxylase 67 promoter [2]. In addition, it was shown that these GABAergic hub cells could single-handedly influence the dynamics of synchronous network events (giant depolarizing potentials). Therefore, by using network theory to infer the functional structure of a developing network from the analysis of its spontaneous dynamics, it was possible to identify and characterize key neurons that were essential for the functional operation of this network. Such an approach would have been nearly impossible, or at least much more difficult, using standard electrophysiological and neuroanatomical techniques. At present, it remains challenging to apply a similar type of analysis in adult networks owing to the sparseness of spontaneous network dynamics in adult slice preparations and the difficulty in imaging neuronal activity from older neurons.

Can hub cells exist in other developing neuronal networks apart from the hippocampus? It is very probable that hub cells exist in other developing networks given the similarity between early network patterns observed in different regions of the developing mammalian brain. For example, network events driven by GABAergic transmission, comparable to the hippocampal giant depolarizing potentials described above, have recently been observed in the developing neocortex [82,102]. Subplate neurons are probable candidates for hub neurons in the developing cortex given that this transient cortical region was recently identified as a 'hub station' [103]. In addition, hippocampal hub cells share several remarkable properties with subsets of subplate neurons, including long distance projections and the ability to drive synchronization in the developing cortical plate [103-105].

What does the finding of hub neurons in the developing hippocampus tell us about the mature hippocampus? For the moment, this question remains a difficult one to address because the morpho-physiological identity of hub cells in adult networks remains to be determined. It is not even known whether or not these cells are only transiently present during early development, as has been reported for subpopulations of hippocampal GABAergic neurons [106]. Theoretical predictions can provide interesting hypotheses regarding the origin of hub nodes (see below). Interestingly, it was recently proposed that the multiple network reorganizations that occur during epileptogenesis would favor the emergence of 'hub neurons', supporting pathological synchronizations [51,107-109]. This is an interesting hypothesis that should be investigated further.

\section{Network growth from a theoretical perspective}

From a theoretical point of view, the process of network growth has always been a very useful model for understanding the emergence of connectivity as well as the general principles and constraints that govern the network structure-function relationship in stable circuits $[54,55,60,110,111]$. In this way, it was proposed that scalefree networks would self-assemble following a 'preferential attachment rule' [60]: scale-free networks arise by the sequential addition of new nodes, and each new coming node has a higher probability to link to highly connected nodes (i.e. hub cells). A possible neurobiological translation of this general network rule applied to the developing hippocampus would be that neurons sequentially receive synaptic inputs from hub neurons as a function of their maturation stage in brain structures, developing into a scale-free mode of organization. Hub neurons would therefore be GABAergic interneurons that developed at early stages during the course of embryogenesis: this hypothesis can be tested in future studies using genetic fate mapping approaches [112]. 


\section{Box 4. Outstanding questions}

- Is the ability of single cells to influence network dynamics always related to the underlying anatomical network topology or are other factors involved?

- Are specific brain tasks associated with specific functional connectivity patterns at the microcircuit level? For example, in vivo analysis of the functional connectivity between place cells in the hippocampus during spatial navigation in awake-behaving rodents could help to address this question.

- Are hub neurons present in other brain regions other than the hippocampus? If so, what is the identity of such neurons?

- Are hub neurons present only in the healthy developing brain, or do they also exist in mature and/or pathological neuronal circuits?

- Under what circumstances will a study of functional/effective connectivity lead to insights about underlying structural connectivity and vice versa?

Interestingly, other theoretical predictions indicate that networks with a broad-tailed degree distribution, such as scale-free networks, can emerge not only by adding connections but also by selectively eliminating them, with a probability that is inversely related to the sum of their first- and second- order connectivity [60,113]. Such a growth-elimination process might well model the developmental cell death/synapse elimination phenomena observed in most developing brain structures. Therefore, theoretical analysis of neuronal network topology can provide many interesting clues for the cellular mechanisms by which connections might be establishing during development. Interestingly, model predictions have also started taking into account the activity of individual nodes in network growth processes [111]. This is essential given that functional connectivity develops in parallel with structural connectivity during brain maturation.

\section{Conclusion}

Structure-function studies of neuronal networks are currently experiencing a very stimulating period because new technologies for probing cells and circuits are providing experimentalists with unprecedented access to neuronal physiology and anatomy at different scales and in the most physiological conditions possible. As larger datasets are obtained, the value of theoretical and computational neuroscience will continue to grow. Hence, theoretical and experimental neuroscientists should cooperate and help to advance our understanding of the influence of connectivity on cortical microcircuit function. This collaboration will be essential in addressing the many outstanding questions concerning structure-function relationships (Box 4). As we have outlined in this review, novel analysis techniques derived from complex network theory can be successfully combined with data from developing microcircuits to provide insight into the important relationship between structure and function in neuronal networks.

\section{Acknowledgments}

We are indebted to Dr Jérôme Epsztejn for his major contribution to the initial stages of preparation of this review and for his critical comments We thank Dr Y. Ben-Ari for critical reading of the review and Dror Kenett for useful suggestions of network analysis software. Research in the Cossart group is supported by grants from the European Research Council (ERC FP7 Young Investigators \#242852), the Fondation pour la Recherche Medicale (Equipe FRM 2008), the Fondation Bettencourt
Schueller, INSERM, the Ville de Marseille and Region Provence Alpes Côte d'Azur, and the Fondation pour la Recherche sur le Cerveau. We also gratefully acknowledge funding support by the Centre National de la Recherche Scientifique (RC), the National Institute of Neurological Disorders and Stroke (Award Number T32NS045540 to SF), the ItalyIsrael Initiative on Integrative Network Neuroscience (PB) and an FP7Intra-European Fellowship for career development (PB).

\section{References}

1 Miles, R. and Wong, R. (1983) Single neurons can initiate synchronized population discharge in the hippocampus. Nature 306, 371-373

2 Bonifazi, P. et al. (2009) GABAergic hub neurons orchestrate synchrony in developing hippocampal networks. Science 326, 1419 1424

3 Brecht, M. et al. (2004) Whisker movements evoked by stimulation of single pyramidal cells in rat motor cortex. Nature 427, 704-710

4 Ellender, T.J. et al. (2010) Priming of hippocampal population bursts by individual perisomatic-targeting interneurons. J. Neurosci. 30, 5979-5991

5 Houweling, A.R. and Brecht, M. (2008) Behavioural report of single neuron stimulation in somatosensory cortex. Nature 451, 65-68

$6 \mathrm{Li}$, C.Y. et al. (2009) Burst spiking of a single cortical neuron modifies global brain state. Science 324, 643-646

7 Molnar, G. et al. (2008) Complex events initiated by individual spikes in the human cerebral cortex. PLoS Biol. 6, e222

8 London, M. et al. (2010) Sensitivity to perturbations in vivo implies high noise and suggests rate coding in cortex. Nature 466, 123-127

9 Claridge-Chang, A. et al. (2009) Writing memories with lightaddressable reinforcement circuitry. Cell 139, 405-415

10 Lichtman, J.W. et al. (2008) A technicolour approach to the connectome. Nat. Rev. Neurosci. 9, 417-422

11 Luo, L. et al. (2008) Genetic dissection of neural circuits. Neuron 57, 634-660

12 Callaway, E.M. (2008) Transneuronal circuit tracing with neurotropic viruses. Curr. Opin. Neurobiol. 18, 617-623

13 Miesenbock, G. (2009) The optogenetic catechism. Science 326, 395-399

14 Scanziani, M. and Hausser, M. (2009) Electrophysiology in the age of light. Nature 461, 930-939

15 Zhang, F. et al. (2007) Circuit-breakers: optical technologies for probing neural signals and systems. Nat. Rev. Neurosci. 8, 577-581

16 Livet, J. et al. (2007) Transgenic strategies for combinatorial expression of fluorescent proteins in the nervous system. Nature $450,56-62$

17 Buzsaki, G. (2004) Large-scale recording of neuronal ensembles. Nat Neurosci. 7, 446-451

18 Chorev, E. et al. (2009) Electrophysiological recordings from behaving animals-going beyond spikes. Curr. Opin. Neurobiol. 19, 513-519

19 Gentet, L.J. et al. (2010) Membrane potential dynamics of GABAergic neurons in the barrel cortex of behaving mice. Neuron 65, 422-435

20 Lee, A.K. et al. (2009) Head-anchored whole-cell recordings in freely moving rats. Nat. Protoc. 4, 385-392

21 Harvey, C.D. et al. (2009) Intracellular dynamics of hippocampal place cells during virtual navigation. Nature 461, 941-946

22 Dombeck, D.A. et al. (2010) Functional imaging of hippocampal place cells at cellular resolution during virtual navigation. Nat. Neurosci. 13, 1433-1440

$23 \mathrm{Jia}, \mathrm{H}$. et al. (2011) In vivo two-photon imaging of sensory-evoked dendritic calcium signals in cortical neurons. Nat. Protoc. 6, 28-35

24 Moser, E.I. and Moser, M.B. (2008) A metric for space. Hippocampus 18, 1142-1156

25 Newman, M.E. (2002) Spread of epidemic disease on networks. Phys Rev. E: Stat. Nonlin. Soft Matter Phys. 66, 016128

26 Salathe, M. et al. (2010) A high-resolution human contact network for infectious disease transmission. Proc. Natl. Acad. Sci. U.S.A. 107, $22020-22025$

27 Dunne,J.A.etal. (2002) Food-web structure and network theory: the role of connectance and size. Proc. Natl. Acad. Sci. U.S.A. 99, 12917-12922

28 Stouffer, D.B. et al. (2006) A robust measure of food web intervality. Proc. Natl. Acad. Sci. U.S.A. 103, 19015-19020

29 Feldt, S. et al. (2009) Functional clustering algorithm for the analysis of dynamic network data. Phys. Rev. E: Stat. Nonlin. Soft Matter Phys. 79,056104 
30 Newman, M.E. (2004) Fast algorithm for detecting community structure in networks. Phys. Rev. E: Stat. Nonlin. Soft Matter Phys. 69, 066133

31 Newman, M.E. (2006) Finding community structure in networks using the eigenvectors of matrices. Phys. Rev. E: Stat. Nonlin. Soft Matter Phys. 74, 036104

32 Rosvall, M. and Bergstrom, C.T. (2008) Maps of random walks on complex networks reveal community structure. Proc. Natl. Acad. Sci. U.S.A. 105, 1118-1123

33 Boccaletti, S. et al. (2006) Complex networks: structure and dynamics. Phys. Rep. 424, 175-308

34 Albert, R. et al. (2000) Error and attack tolerance of complex networks. Nature 406, 378-382

35 Chu-Shore, J. et al. (2010) Power law versus exponential state transition dynamics: application to sleep-wake architecture. PLoS ONE 5, e14204

36 Clauset, A. et al. (2009) Power-law distributions in empirical data. SIAM Rev. 51, 661-703

37 Watts, D.J. and Strogatz, S.H. (1998) Collective dynamics of 'smallworld' networks. Nature 393, 440-442

38 Rubinov, M. and Sporns, O. (2010) Complex network measures of brain connectivity: uses and interpretations. Neuroimage 52,1059 1069

39 Sporns, O. and Kotter, R. (2004) Motifs in brain networks. PLoS Biol. 2 , e369

40 Achard, S. et al. (2006) A resilient, low-frequency, small-world human brain functional network with highly connected association cortical hubs. J. Neurosci. 26, 63-72

41 Honey, C.J. et al. (2010) Can structure predict function in the human brain? Neuroimage 52, 766-776

42 Ponten, S.C. et al. (2007) Small-world networks and epilepsy: Graph theoretical analysis of intracerebrally recorded mesial temporal lobe seizures. Clin. Neurophysiol. 118, 918-927

$43 \mathrm{Yu}$, S. et al. (2008) A Small World of Neuronal Synchrony. Cereb. Cortex 18, 2891-2901

44 Bullmore, E. and Sporns, O. (2009) Complex brain networks: graph theoretical analysis of structural and functional systems. Nat. Rev. Neurosci. 10, 186-198

45 Klausberger, T. et al. (2003) Brain-state- and cell-type-specific firing of hippocampal interneurons in vivo. Nature 421, 844-848

46 Aertsen, A.M. et al. (1989) Dynamics of neuronal firing correlation: modulation of 'effective connectivity'. J. Neurophysiol. 61, 900-917

47 Friston, K. (2009) Causal modelling and brain connectivity in functional magnetic resonance imaging. PLoS Biol. 7, e33

48 Arenas, A. et al. (2008) Synchronization in complex networks. Phys. Rep. 469, 93-153

49 Barahona, M. and Pecora, L.M. (2002) Synchronization in small-world systems. Phys. Rev. Lett. 89, 054101

50 Feldt, S. et al. (2010) Memory formation: from network structure to neural dynamics. Philos. Trans. A: Math. Phys. Eng. Sci. 368, 22512267

51 Morgan, R.J. and Soltesz, I. (2008) Nonrandom connectivity of the epileptic dentate gyrus predicts a major role for neuronal hubs in seizures. Proc. Natl. Acad. Sci. U.S.A. 105, 6179-6184

52 Wang, X.F. and Chen, G.R. (2002) Synchronization in small-world dynamical networks. Int. J. Bifurcat. Chaos 12, 187-192

53 Kumar, A. et al. (2010) Spiking activity propagation in neuronal networks: reconciling different perspectives on neural coding. Nat. Rev. Neurosci. 11, 615-627

54 Fuchs, E. et al. (2007) Coemergence of regularity and complexity during neural network development. Dev. Neurobiol. 67, 1802-1814

55 Fuchs, E. et al. (2009) The formation of synchronization cliques during the development of modular neural networks. Phys. Biol. 6, 036018

56 Percha, B. et al. (2005) Transition from local to global phase synchrony in small world neural network and its possible implications for epilepsy. Phys. Rev. E: Stat. Nonlin. Soft Matter Phys. 72, 031909

57 Roxin, A. et al. (2004) Self-sustained activity in a small-world network of excitable neurons. Phys. Rev. Lett. 92, 198101

58 Watt, A.J. et al. (2009) Traveling waves in developing cerebellar cortex mediated by asymmetrical Purkinje cell connectivity. Nat. Neurosci. 12, 463-473

59 Amaral, L.A. et al. (2000) Classes of small-world networks. Proc. Natl. Acad. Sci. U.S.A. 97, 11149-11152
60 Barabasi, A.L. and Albert, R. (1999) Emergence of scaling in random networks. Science 286, 509-512

61 Buzsaki, G. et al. (2004) Interneuron Diversity series: Circuit complexity and axon wiring economy of cortical interneurons. Trends Neurosci. 27, 186-193

62 Grinstein, G. and Linsker, R. (2005) Synchronous neural activity in scale-free network models versus random network models. Proc. Natl. Acad. Sci. U.S.A. 102, 9948-9953

63 Lubenov, E.V. and Siapas, A.G. (2009) Hippocampal theta oscillations are travelling waves. Nature 459, 534-539

64 Chen, B.L. et al. (2006) Wiring optimization can relate neuronal structure and function. Proc. Natl. Acad. Sci. U.S.A. 103, 4723-4728

65 Barabasi, A.L. and Oltvai, Z.N. (2004) Network biology: understanding the cell's functional organization. Nat. Rev. Genet. 5, 101-113

66 Bassett, D.S. et al. (2010) Efficient physical embedding of topologically complex information processing networks in brains and computer circuits. PLoS Comput. Biol. 6, e1000748

67 Voges, N. et al. (2010) A modeler's view on the spatial structure of intrinsic horizontal connectivity in the neocortex. Prog. Neurobiol. 92, 277-292

68 Sumbre, G. et al. (2008) Entrained rhythmic activities of neuronal ensembles as perceptual memory of time interval. Nature 456, 102-106

69 Macosko, E.Z. et al. (2009) A hub-and-spoke circuit drives pheromone attraction and social behaviour in C. elegans. Nature 458, 1171-1175

70 Shang, Y. et al. (2007) Excitatory local circuits and their implications for olfactory processing in the fly antennal lobe. Cell 128, 601-612

71 Laurent, G. (2002) Olfactory network dynamics and the coding of multidimensional signals. Nat. Rev. Neurosci. 3, 884-895

72 Jortner, R.A. et al. (2007) A simple connectivity scheme for sparse coding in an olfactory system. J. Neurosci. 27, 1659-1669

73 Sur, M. and Rubenstein, J.L. (2005) Patterning and plasticity of the cerebral cortex. Science 310, 805-810

$74 \mathrm{Yu}$, Y.C. et al. (2009) Specific synapses develop preferentially among sister excitatory neurons in the neocortex. Nature 458, 501-504

75 Batista-Brito, R. and Fishell, G. (2009) The developmental integration of cortical interneurons into a functional network. Curr. Top. Dev. Biol. 87, 81-118

76 Butt, S.J. et al. (2005) The temporal and spatial origins of cortical interneurons predict their physiological subtype. Neuron 48, 591-604

77 Allene, C. and Cossart, R. (2009) Early NMDA receptor-driven waves of activity in the developing neocortex: physiological or pathological network oscillations? J. Physiol. 588, 83-91

78 Ben Ari, Y. (2001) Developing networks play a similar melody. Trends Neurosci. 24, 353-360

79 Blankenship, A.G. and Feller, M.B. (2010) Mechanisms underlying spontaneous patterned activity in developing neural circuits. Nat. Rev. Neurosci. 11, 18-29

80 Moody, W.J. and Bosma, M.M. (2005) Ion channel development, spontaneous activity, and activity-dependent development in nerve and muscle cells. Physiol. Rev. 85, 883-941

81 Spitzer, N.C. (2006) Electrical activity in early neuronal development. Nature 444, 707-712

82 Allene, C. et al. (2008) Sequential generation of two distinct synapsedriven network patterns in developing neocortex. J. Neurosci. 28 , 12851-12863

83 Garaschuk, O. et al. (2000) Large-scale oscillatory calcium waves in the immature cortex. Nat. Neurosci. 3, 452-459

$84 \mathrm{McCabe}$, A.K. et al. (2007) Roles of glutamate and GABA receptors in setting the developmental timing of spontaneous synchronized activity in the developing mouse cortex. Dev. Neurobiol. 67, 15741588

85 Rochefort, N.L. et al. (2009) Sparsification of neuronal activity in the visual cortex at eye-opening. Proc. Natl. Acad. Sci. U.S.A. 106, 15049-15054

86 Adelsberger, H. et al. (2005) Cortical calcium waves in resting newborn mice. Nat. Neurosci. 8, 988-990

87 Khazipov, R. et al. (2004) Early motor activity drives spindle bursts in the developing somatosensory cortex. Nature 432, 758-761

88 Leinekugel, X. et al. (2002) Correlated bursts of activity in the neonatal hippocampus in vivo. Science 296, 2049-2052 
89 Yang, J.W. et al. (2009) Three patterns of oscillatory activity differentially synchronize developing neocortical networks in vivo. J. Neurosci. 29, 9011-9025

90 Bettencourt, L.M. et al. (2007) Functional structure of cortical neuronal networks grown in vitro. Phys. Rev. E: Stat. Nonlin. Soft Matter Phys. 75, 021915

91 Eytan, D. and Marom, S. (2006) Dynamics and effective topology underlying synchronization in networks of cortical neurons. $J$. Neurosci. 26, 8465-8476

92 Srinivas, K.V. et al. (2007) Small-world network topology of hippocampal neuronal network is lost, in an in vitro glutamate injury model of epilepsy. Eur. J. Neurosci. 25, 3276-3286

93 Kerchner, G.A. and Nicoll, R.A. (2008) Silent synapses and the emergence of a postsynaptic mechanism for LTP. Nat. Rev. Neurosci. 9, 813-825

94 Crepel, V. et al. (2007) A parturition-associated nonsynaptic coherent activity pattern in the developing hippocampus. Neuron 54, 105-120

95 Dupont, E. et al. (2006) Rapid developmental switch in the mechanisms driving early cortical columnar networks. Nature 439, 79-83

96 Ben-Ari, Y. et al. (2007) GABA: a pioneer transmitter that excites immature neurons and generates primitive oscillations. Physiol. Rev. $87,1215-1284$

97 Wang, D.D. and Kriegstein, A.R. (2008) GABA regulates excitatory synapse formation in the neocortex via NMDA receptor activation. $J$. Neurosci. 28, 5547-5558

98 Blankenship, A.G. et al. (2009) Synaptic and extrasynaptic factors governing glutamatergic retinal waves. Neuron 62, 230-241

99 Segerstrale, M. et al. (2010) High firing rate of neonatal hippocampal interneurons is caused by attenuation of afterhyperpolarizing potassium currents by tonically active kainate receptors. J. Neurosci. $30,6507-6514$

100 Hagmann, P. et al. (2010) White matter maturation reshapes structural connectivity in the late developing human brain. Proc. Natl. Acad. Sci. U.S.A. 107, 19067-19072

101 Supekar, K. et al. (2010) Development of functional and structural connectivity within the default mode network in young children. Neuroimage 52, 290-301
102 Rheims, S. et al. (2008) Excitatory GABA in rodent developing neocortex in vitro. J. Neurophysiol. 100, 609-619

103 Kanold, P.O. and Luhmann, H.J. (2010) The subplate and early cortical circuits. Annu. Rev. Neurosci. 33, 23-48

104 Tamamaki, N. and Tomioka, R. (2010) Long-Range GABAergic Connections Distributed throughout the Neocortex and their Possible Function. Front. Neurosci. 4, 202

105 Voigt, T. et al. (2001) Synchronous oscillatory activity in immature cortical network is driven by GABAergic preplate neurons. $J$. Neurosci. 21, 8895-8905

106 Super, H. et al. (1998) Involvement of distinct pioneer neurons in the formation of layer-specific connections in the hippocampus. $J$. Neurosci. 18, 4616-4626

107 Case, M. and Soltesz, I. (2009) Discreet charm of the GABAergic bourgeoisie: superconnected cells conduct developmental symphonies. Neuron 64, 780-782

108 Thind, K.K. et al. (2010) Initial loss but later excess of GABAergic synapses with dentate granule cells in a rat model of temporal lobe epilepsy. J. Comp. Neurol. 518, 647-667

109 Zhang, W. et al. (2009) Surviving hilar somatostatin interneurons enlarge, sprout axons, and form new synapses with granule cells in a mouse model of temporal lobe epilepsy. J. Neurosci. 29, 14247-14256

110 Kaiser, M. et al. (2009) A simple rule for axon outgrowth and synaptic competition generates realistic connection lengths and filling fractions. Cereb. Cortex 19, 3001-3010

111 Stam, C.J. et al. (2010) Emergence of Modular Structure in a LargeScale Brain Network with Interactions between Dynamics and Connectivity. Front. Comput. Neurosci. 4

112 Miyoshi, G. and Fishell, G. (2006) Directing neuron-specific transgene expression in the mouse CNS. Curr. Opin. Neurobiol. 16, 577-584

113 Salathe, M. et al. (2005) The evolution of network topology by selective removal. J. R. Soc. Interface 2, 533-536

114 Wittner, L. and Miles, R. (2007) Factors defining a pacemaker region for synchrony in the hippocampus. J. Physiol. 584, 867-883

115 Buckner, R.L. et al. (2009) Cortical hubs revealed by intrinsic functional connectivity: mapping, assessment of stability, and relation to Alzheimer's disease. J. Neurosci. 29, 1860-1873 